\title{
Consideraciones orales del síndrome de Kawasaki: Descripción de un caso clínico
}

\section{Oral considerations of Kawasaki syndrome: A case report}

\author{
Figueiredo MC*, Pires PDS**, Faustino-Silva DD***, Cernicchiaro MO****, \\ Squef $\mathrm{R}^{* * * * * *}$
}

\section{RESUMEN}

El objetivo del presente estudio es presentar un caso clínico y revisar la literatura existente sobre el Síndrome de Kawasaki (SK) tan frecuente en pacientes con necesidades especiales. Las características clínicas de esta patología se observan ya en niños muy jóvenes, en la gran mayoría de los casos menores de 5 años de edad. Esta es una patología que puede llevar al óbito, pues revela un compromiso cardíaco importante. Hay señales y síntomas en la cavidad oral en la fase aguda de la enfermedad, como la lengua de "frambuesa", eritema con fisura labial y eritema en la mucosa oral, siendo importante que el dentista tenga el conocimiento de esta patología y encamine el paciente para tratamiento médico con urgencia. Se observó en este caso una característica peculiar, que es la retención prolongada de la dentición temporaria. Una vez diagnosticado y tratado, el conocimiento del Síndrome de Kawasaki permite al dentista planear con seguridad un tratamiento dentario a largo plazo. Con eso puede evitarse que los dientes permanentes erupcionen ectópicamente, así como también mantener una profilaxis bucal de rutina, preservando la salud oral de los pacientes portadores de este síndrome.

Palabras clave: Síndrome de Kawasaki, síndrome mucocutáneo linfonodular, cavidad oral, paciente con necesidades especiales, aneurisma coronario.

\section{SUMMARY}

The aim of this study is to review what literature presents about Kawasaki Disease (KD), common in patients with special needs. Clinical evidence of this pathology may be observed in young children, who are usually less than five years old. This pathology may lead patients to death since it involves serious heart conditions. There are signs and symptoms in the oral cavity during the acute phase, for example "strawberry" tongue, red lesions with lips fissure and red lesions of oral mucous. Therefore it is important for the dentist to know about this pathology in order to send the patient to urgent medical treatment. A peculiar characteristic was observed in this case, long term retention of temporary dentition. Once the disease has been diagnosed and treated, it

* Profesora Doctora de la Disciplina de Odontopediatría y del Curso de Extensión Universitaria Atención de Pacientes Portadores de Necesidades especiales. Facultad de Odontología. Universidad Federal de Río Grande do Sul. Brasil.

** Odontóloga, alumna interna del Curso de Extensión Universitaria Atención de Pacientes Portadores de Necesidades Especiales de la Facultad de Odontología. Universidad Federal de Río Grande do Sul. Brasil.

*** Especialista en Salud Colectiva, maestría en Clínicas Odontológicas: Odontopediatría. Facultad de Odontología. Universidad Federal de Río Grande do Sul. Brasil.

**** Académico. Facultad de Odontología. Universidad Federal de Río Grande do Sul. Brasil.

***** Odontóloga, ex-alumna del Curso de Extensión Universitaria Bebé Clínica de la Facultad de Odontología. Universidad Federal de Río Grande do Sul. Brasil. 
allows dentists to plan long term and safe treatment. That may stop permanent dentition to go through an ectopic outbreak, besides keeping a custom oral prophylaxis and preserving these patients' oral health.

Key words: Kawasaki disease, mucocutaneous lymph node syndrome, oral cavity, patients with special needs, heart endanger.

Fecha de recepción: 15 de abril de 2009.

Aceptado para publicación: 11 de junio de 2009.

Figueiredo MC, Pires PDS, Faustino-Silva DD, Cernicchiaro MO, Squef R. Consideraciones orales del síndrome de Kawasaki: Descripción de un caso clínico. Av. Odontoestomatol 2010; 26 (4): 203-207.

\section{INTRODUCCIÓN}

El Síndrome de Kawasaki (SK) fue descrito por primera vez en 1967 por el Dr. Tomisaki Kawasaki, que describió 50 casos en 6 años, en el Centro Médico de la Cruz Roja en Tokio (1). Esta enfermedad ha sido objeto de interés epidemiológico, clínico y de laboratorio (2). Es una enfermedad sistémica, de origen desconocida, autolimitada, de pequeños y medianos vasos (3), siendo que las secuelas cardíacas tal como el aneurisma coronario (Fig. 1), estenosis coronaria e infarto del miocardio son los problemas más serios (4). Estudios recientes describen que el factor inmunológico puede ser una de las causas de esta enfermedad (5).

En Japón la enfermedad ha aumentado considerablemente con una frecuencia anual de 5.000 a 6.000

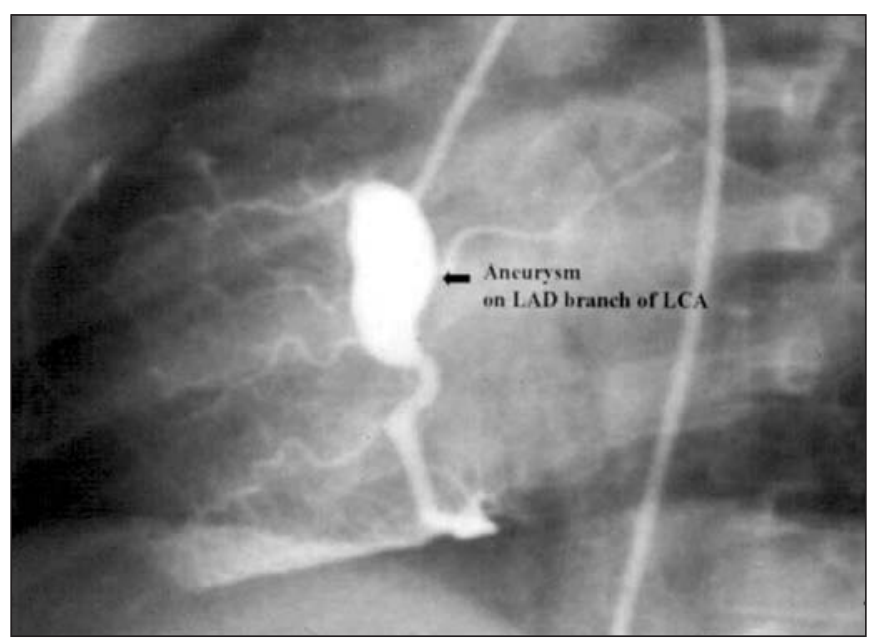

Fig. 1. Aneurisma coronario. Fuente: Kim D.S. (2006). casos/año, con característica endémica (3), epidemias registradas en los años 1979, 1982 y 1988, sugiriendo que el Síndrome de Kawasaki puede tener también un origen viral (1). Hay registros que evidencian ser una enfermedad de etiología infecciosa, causada por toxinas bacterianas que actúan en los linfocitos, promoviendo alteración de los receptores de las células (6). Debido al severo compromiso que este síndrome acarrea al sistema cardíaco, es de suma importancia establecer su diagnóstico precozmente (1).

Su principal característica es una vasculitis generalizada, lo que ocurre en el $40 \%$ de los casos. Este daño coronario lleva a la formación de aneurismas, isquemia del miocardio, angina e infarto (5). Por más que la afectación del Sistema Nervioso Central no sea frecuente, fueron descritos en la literatura 13 casos de deficiencia auditiva neurosensorial durante la evolución de esta enfermedad. Ésta es también una complicación grave poco mencionada en la literatura que viene a reforzar la recomendación de cautela en la evaluación de los pacientes (3). En los pacientes diagnosticados con pérdida auditiva neurosensorial, asociada a la SK, la parálisis facial y ataxia son otras manifestaciones neurológicas, evidenciadas en el diagnóstico (3).

La prevalencia mayor de esta enfermedad es en niños menores de cinco años y mayores de dos años (2, 4-7). Es raro en niños con más de 12 años, pero actualmente han sido descritos estudios en adultos (6), en estos, el primer diagnóstico es establecido como shock tóxico, pero hoy se acepta su presencia en este tipo de edad (1). La mortalidad es igualmente más frecuente en niños menores, siendo más común en niños que en niñas, en una proporción de 
1,4:1(1). Es más frecuente en los pueblos orientales, principalmente el Japón (6), pero se ha descrito con cierta frecuencia en Venezuela (8). Por ser la etiología del síndrome todavía no muy bien conocida, debe tener su diagnóstico diferenciando de las infecciones por staphylococcus, streptococcus, Sarampión, Síndrome de Steven's Johnson, reacción medicamentosa y artritis reumatoide juvenil (8), Leptospirosis, fiebre de las montañas rocosas (6).

Los criterios para el establecimiento del diagnóstico del Síndrome de Kawasaki pueden ser divididos en tres fases (1):

1. Fase febril aguda (de 7 a 14 días): Fiebre alta en torno de 40 grados, asociada a una infección conjuntiva, edema y eritema de pies y manos, diarrea y disfunción hepática.

2. Fase subaguda (de 10 a 24 días): Los señales de fiebre, exantema y linfadenopatía desaparecen y persiste la irritabilidad, la anorexia y la infección conjuntiva. En esta fase se presentan artritis, disfunción miocárdica y trombosis.

3. Fase de convalecencia (de 6 a 8 semanas): Después del inicio de la enfermedad, donde desaparecen todos los síntomas y persisten las alteraciones cardíacas. Es la fase de riesgo de la enfermedad coronaria.

Es importante al establecer el diagnóstico de Síndrome de Kawasaki que el paciente presente al menos cuatro de los seis síntomas o señales clínicas principales citados a continuación $(2,8)$ :

- Infección de la conjuntiva.

- Alteración de la mucosa oral.

- Exantemas en el tronco.

- Fiebre alta.

- Ganglio cervical palpable, normalmente unilateral, no supurativo, que desaparece rápidamente.

- Edema y eritema de manos y pies.

- Exclusión de enfermedades: infección por staphylococcus o streptococcus, Sarampión, Leptospirosis, Artritis Reumatoide Juvenil, reacción medicamentosa, Síndrome de Steven's Johnson.

Hay señales y síntomas en la cavidad oral en la fase aguda de la enfermedad, como la lengua de "frambuesa" (Fig. 2), eritema con fisura labial y eritema en la mucosa oral, siendo importante que el dentista tenga el conocimiento de esta patología y encamine el paciente para tratamiento médico con urgencia.

\section{DESCRIPCIÓN DEL CASO CLÍNICO}

El paciente, de sexo masculino, nacido en año de 1996, con historia de prenatal y postnatal normales. Llegó al hospital de su ciudad con cuatro años y siete meses presentando fiebre alta y después de 21 días fue encaminado por el equipo médico al Hospital de referencia en la capital cercana, por no responder positivamente a ningún tratamiento, persistiendo el cuadro febril. Al llegar al Hospital, el paciente presentaba el siguiente cuadro clínico: fiebre, edema con aumento de un ganglio de la región cervical derecha, eritema de la conjuntiva sin secreción ocular, eritema de la mucosa oral, edema y fisura de los labios, distensión abdominal, vómito, diarrea, hematuria, edema de las extremidades, rostro y región escrotal, descamación de pies y manos. Se administró Despacilina y Pen G Benzatina y, después de cinco días, se obtuvo éxito en relación a la adenomegalia, por más de que el cuadro febril persistía.

Los exámenes laboratoriales realizados mostraron: PCR (Proteína C Reactiva) y VSG (Velocidad de Segmentación Globular), que son marcadores inflamatorios y infecciosos aumentados, y demás exámenes

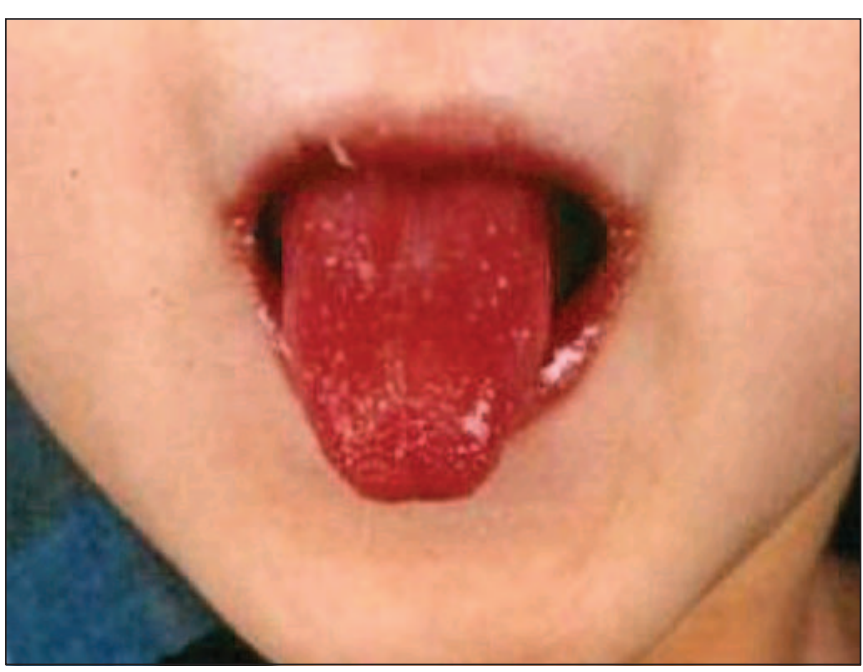

Fig. 2. Lengua de "frambuesa" y eritema con fisura labial. Fuente: Kim D.S. 
normales. El examen de eco cardiografía presentó: aneurismas gigantes, ectasia coronaria, derrame pericardio y trombo no oclusivo en aneurisma de circunfleja. Entonces, a partir de esto se estableció el diagnóstico de Síndrome de Kawasaki y la atención médica para esta patología.

Posteriormente el paciente fue enviado a la Clínica Odontológica para Pacientes Portadores de Necesidades Especiales de la Facultad de Odontología de la Universidad Federal de Río Grande del Sur UFRGS, Brasil, con el objetivo de hacer un informe de su salud bucal. Entonces, recibió un tratamiento educativopreventivo para mantener las buenas condiciones orales, libre de caries y enfermedades de las encías.

Pero a lo largo del tiempo, se ha observado la retención prolongada de la dentición temporaria, pues el paciente ya se encontraba con 12 años y sus molares temporarios no presentaban los indicios de movilidad que llevarían al cambio por los permanentes.

Entonces, debido a la observación clínica de la retención temporaria prolongada se determinó la necesidad de la extracción de los elementos dentarios 54, 64, 74, $84,55,65,75$ y 85 en orden cronológico. Previamente a los procedimientos quirúrgicos, se realizó una profilaxis antimicrobiana para endocarditis bacteriana con $2 \mathrm{~g}$ de Amoxicilina, una hora antes del procedimiento. Para estos procedimientos, fue utilizado anestésico con vasoconstrictor (lidocaína con adrenalina).

\section{DISCUSIÓN}

El compromiso cardíaco, en especial, la vasculitis coronaria está entre las principales complicaciones de el Síndrome de Kawasaki (SK) (3). Su origen se ha discutido ampliamente, desde causas infecciosas por bacterias (6), por virus (1) e inmunológicas (5). Por acarrear afectación cardíaca amplia es fundamental que su diagnóstico se establezca precozmente (1), anticipándonos a la formación de isquemia del miocardio, aneurismas, angina e infarto (5). Estas alteraciones siempre deben observarse por el dentista cuando procedimientos invasivos o quirúrgicos deban ser practicados en pacientes con SK. Por eso, en el caso descrito se utilizó la profilaxis antimicrobiana previa según las recomendaciones de la academia americana de cardiología, con el objetivo de prevenir la endocarditis bacteriana, incluso cuando el riesgo sea bajo para los pacientes con SK $(9,10)$.

El uso de anestésico local también es un tema preocupante para el dentista durante la atención con compromiso cardíaco. Por otro lado, las repercusiones sistémicas y cardiovasculares del uso de vasoconstrictores locales para procedimientos dentarios son muy limitadas según algunos estudios (11-15). Por esto, pueden ser usados anestésicos con vasoconstrictor en pacientes con la SK, desde que sean atendidas las dosis apropiadas para su edad y que las condiciones clínicas cardiovasculares estén controladas. Aparte de esto, es importante que el dentista no se olvide del manejo comportamental del paciente, o sea, tratarlo con tranquilidad y atención para con eso disminuir los índices de ansiedad y dolor a lo largo del procedimiento.

Después de las extracciones, era posible percibir la proximidad con el diente permanente de cada temporario extraído. Este hecho evidencia la tardanza de la retención temporaria que fue encontrado en este caso. No se han encontrado descripciones de casos o estudios sobre las manifestaciones orales del SK, entonces, como no hay parámetros en el literatura, no se puede afirmar que la retención temporaria es una consecuencia del síndrome. Los riesgos de mantener los temporarios tardíamente son la anquilosis o la erupción ectópica de los permanentes y consecuentemente, alteraciones de oclusión considerables.

En menor escala, pero actualmente citado en la literatura, ocurre compromiso del Sistema Nervioso Central, ocasionando la deficiencia auditiva neurosensorial, durante el proceso evolutivo de la enfermedad (3). El paciente de este caso no presenta tal alteración, pero es importante la observación de las respuestas auditivas, y en el caso de presentar deficiencias el dentista deberá estar apto para sostener una comunicación no verbal con el paciente.

\section{CONCLUSIÓN}

Los resultados de esta presentación clínica indican la importancia del diagnóstico precoz en el Síndrome de Kawasaki. Sus manifestaciones bucales en la 
fase aguda exigen que dentistas cirujanos tengan el conocimiento de este síndrome, una vez que este profesional puede realizar, en primera instancia, su correcto diagnóstico. Aparte de esto, el estudio odontológico del paciente requiere cuidados específicos del síndrome, así como un buen mantenimiento de higiene y salud bucal se tornan de extrema importancia en la prevención de comorbilidades asociadas al síndrome.

\section{BIBLIOGRAFÍA}

1. Jaramillo CMG. Enfermedad de Kawasaki. Revista CES de Medicina 2000; 14(2):26-36.

2. Banfi AP. Enfermedad de Kawasaki. Rev Chil Pediatr 2001; 72(6):487-95.

3. Silva CHM, Roscoe ICRG, Fernandes KP, Novaes RM Lázari CS. Deficiência auditiva neurossensorial associada à doença de Kawasaki. J Pediatr 2002; 78(1):71-4.

4. Nakamara Y. Aso E. Yashiro M. Uehara R. Watanabe M. Oki I. Yanagawa H. Mortality Among Persons with a History of Kawasaki Disease in Japan. Circ J 2008; 72(1):134-8.

5. Ferrán RF, Gómez OR, Acuña JAR, Placencia CC. Enfermedad de Kawasaki: a propósito de un caso. Rev Cubana Pediatr 2000;72(3):220-4.

6. Carvajal CC. Enfermedad de Kawasaki. Rev Cubana Pediatr [periódico en la Internet] 2003 [citado 2009 Feb 03]; 75(3). Disponible en: http:/ /scielo.sld.cu/scielo.php?script=sci_arttextEpid = S0034-75312003000300009Elng $=$ esEnrm $=$ iso

7. Schonhaut LB, Herrera P, Acevedo K, Álvarez P. Enfermedad de Kawasaki en el Hospital Roberto del Río: análisis clínico epidemiológico. Rev Chil Pediatr 2001;72(4):319-27.

8. De la Parte MA. Terán L.E. Barreto N. Rodriguez S. Contreras F. Enfermedad de Kawasaki-Report de un caso. BSVM 2000;20(1):01-01.
9. Gutiérrez JL, Bagán JV, Bascones A, Llamas R, Llena J, Morales A, et al. Consensus document on the use of antibiotic prophylaxis in dental surgery and procedures. Med Oral Patol Oral Cir Bucal 2006;11(2):E188-205.

10. Carrión AB. Profilaxis de la endocarditis bacteriana. Med Oral Patol Oral Cir Bucal 2004;9 (Suppl):S37-51.

11. Caceres MTF, Ludovice ACPP, Brito FS, Darrieux FC, Neves RS, Scanavacca MI et al. Efeito de anestésicos locais com e sem vasoconstritor em pacientes com arritmias ventriculares. Arq Bras Cardiol 2008;91(3):142-7.

12. Pérusse R, Goulet JP, Turcotte JY. Contrandications to vasoconstritors in dentistry: part I. Cardiovascular Diseases. Oral Surg Oral Med Oral Pathol 1992;74(5):679-86.

13. Pérusse R, Goulet JP, Turcotte JY. Contraindications to vasoconstrictors in dentistry: part II. Hyperthyroidism, diabetes, sulfite sensitivity, corticodependent asthma, and pheochromocytoma. Oral Surg Oral Med Oral Pathol 1992; 74(5):687-91.

14. Conrado VCLS, Andrade J, Angelis GAMC, Andrade ACP, Timerman L, Andrade MM et al. Cardiovascular effects of local anesthesia with vasoconstrictor during dental extraction in coronary patients. Arq Bras Cardiol 2007;88(5):446-52.

15. Neves SR, Neves ILI, Giorgi DMA, Grupi CJ, César LAM. Hueb W, Grinberg M. Effects of epinephrine in local anesthesia in patients with coronary artery disease. Arq Bras Cardiol 2007;88(5):482-7.

16. Kim DS. Kawasaki Disease. Yonsei Medical Journal 2006;47(6):759-72.

\section{CORRESPONDENCIA}

Dra. MC Figueiredo

mcf1958@gmail.com 